\title{
THE TRUE NATURE OF NON-LINEAR DYNAMIC EFFECTS: A METHODOLOGY TO THE RESCUE OF IMPRECISE THEORY
}

\author{
Ralitza Nikolaeva, ISCTE-University Institute of Lisbon, Portugal
}

\begin{abstract}
There are two major problems with the fundamental advancement of empirical research in the management sciences - there is very little theory regarding temporal effects and methodologies to measure complex relationships are not well known. The goal of the study is to address these concerns with an example of a firm survival study. First, theoretical arguments based on the notions of imprinting of initial conditions and inertia explain why survival determinant effects would change as firms age. The mechanisms of imprinting and inertia affect firms' awareness of which capabilities need more investment. As capabilities change over time, firms' interaction with the environment changes as well. Further, organizational capabilities and resources are contextual. Hence, it is the firm interaction with the environment that determines its success and ultimately its mortality. Because this interaction is dynamic and because firms evolve, even constant factors may affect an organization's mortality in a different way at different stages in the organization's life. The second goal of the study is to improve the state of the art method of estimating the Cox proportional hazards model in management. This type of model has a good tradition of utilization in the marketing and management literature. Yet, although, it has a pronounced methodological requirement for the possible inclusion of dynamics, the assumption of proportional hazards is rarely tested. Using available methods mostly from the medical literature, the study emphasizes the importance of testing the proportionality assumption and demonstrates a method to alleviate the problem of non-proportionality based on fractional polynomials. The flexible method accounts for time-varying effects by interacting covariates with fractional polynomials of time. The study is set in the online retailing segment, which offers a good context for testing the time-variability of various effects on survival probabilities. Being characterized as a turbulent environment and as a recent case of a market bubble, it is a telling example of the fault in assumptions of constant effects. Compared to a constant effects model, the dynamic model provides a better fit and complies with the theoretical predictions. Based on the findings, it is recommended that researchers incorporate a check of the proportionality assumption as a routine procedure in the estimation of proportional hazards regressions.
\end{abstract}

\section{References}

Available upon request. 